\title{
AFM Investigation of the Organization of Actin Bundles Formed by Actin-Binding Proteins
}

\author{
Jamie L. Gilmore, Masahiro Kumeta, Kunio Takeyasu
}

Graduate School of Biostudies, Kyoto University, Kyoto, Japan.

Email: takeyasu@lif.kyoto-u.ac.jp

Received June 21 $1^{\text {st }}$ 2013; revised July 20 $0^{\text {th }}, 2013$; accepted August $6^{\text {th }}, 2013$

Copyright (c) 2013 Jamie L. Gilmore et al. This is an open access article distributed under the Creative Commons Attribution License, which permits unrestricted use, distribution, and reproduction in any medium, provided the original work is properly cited.

\begin{abstract}
AFM is a powerful technique for revealing the morphological features of various biological systems at high resolution. However, one of the complications of AFM is that samples must be attached to a flat surface in order to obtain images. This often requires the development of specialized methods depending on the sample which is being used. In this study, we developed a novel technique to image actin bundles on the mica surface. Using this technique, we were able to image molecular assemblies of F-actin with two actin remodeling proteins: $\alpha$-actinin and Caprice. High resolution AFM images of F-actin fibers and bundle organization depicted two different types of molecular assemblies: F-actin bundles forming an elongated "zipper" structure in the presence of $\alpha$-actinin, and bundles forming a perpendicularly crossing the mesh structure in the presence of Caprice.
\end{abstract}

Keywords: F-Actin; Caprice; $\alpha$-Actinin

\section{Introduction}

The biological applications of atomic force microscopy (AFM) [1] were realized immediately after the instrument was invented in 1986 [2]. Since AFM was useful for examining the surface properties of relatively solid specimens, it was initially used in the field of material sciences. The physical properties of DNA, proteins, lipids and carbohydrates have been extensively studied by using AFM [3-6].

There have been a number of limitations associated with AFM technology. However, the development of new scanning methods [7] and cantilever production [8] has facilitated the applications of AFM for studying biological macromolecules in physiological conditions. The most fascinating technical endeavor has been the invention of high-speed AFM by Ando et al., which has enabled one to capture the motion of DNA [9-12] and proteins [13-17] and to monitor enzymatic reactions [9,11, $18]$ in solution.

However, one of the inherent limitations associated with AFM other than the instrument technology is the specimen preparation procedure, which needs to be taken into account the physicochemical properties of the specimen against substrates such as mica and glass surfaces. Here we describe procedures to image the most abundant protein in the cell: actin. We developed specimen preparation procedures for imaging single filamentous actin (F-actin) and actin-binding protein-promoted F-actin bundles. AFM imaging of these samples provided high resolution images of bundle nanostructures in addition to the ultrastructure of actin networks. The data suggest that F-actin bundles form an elongated "zipper" structure when the $\alpha$-actinin bundling protein is added and the bundles form a perpendicularly crossing meshwork structure when the Caprice protein is added. These structures provide insight into the molecular mechanisms of forming distinct actin-based structures by different actin-binding proteins.

\section{Materials and Methods}

\subsection{Chemicals and G-Actin}

All chemicals used were first grade from Sigma and Nacalai Tesque. The non-muscle human actin was obtained from Cytoskeleton, Inc. (APHL99).

\subsection{Actin-Binding Proteins}

The $\alpha$-actinin from rabbit skeletal muscle, which is a mixture of the actinin-1, 2, 3, and 4 isoforms, was obtained from Cytoskeleton, Inc. (AT01). Caprice (C19orf21 
actin bundling protein in characteristic epithelial cells) [19,20] (NM_173481) is a gene product of human C19orf21, also called MISP (mitotic interactor and substrate of Plk1) [21]. The full-length Caprice expressed in Eschelichia coli cannot be purified due to its "scaffold" nature and instability in the host cell. The actin-bundling domain exists in the C-terminal region of Caprice. Therefore, the 3'-region of the cDNA encoding the C-terminus half of human Caprice, amino acid residues (352-680), referred to as Caprice hereafter, was amplified by PCR from a HeLa cDNA pool, which was reverse transcribed by SuperScript II reverse transcriptase (Invitrogen) from the total RNA extracted by RN-easy RNA extraction kit (Qiagen). The cDNA was cloned into pGEX-6P vector (Clontech) and expressed in BL21RIL E. coli. GST-fusion proteins were purified with glutathione-conjugated sepharose (GE) and the GST-tag was excised by PreScission protease (GE).

\subsection{F-Actin Fiber and Bundle Preparation}

The F-action was prepared from $20 \mu \mathrm{M}$ (900 ng/ $\mu \mathrm{L}$ ) $\mathrm{G}$-action in polymerization buffer $(5 \mathrm{mM}$ Tris- $\mathrm{HCl} \mathrm{pH}$ 8.0, $50 \mathrm{nN} \mathrm{KCl,} 0.2 \mathrm{mM} \mathrm{CaCl}_{2}, 2 \mathrm{mM} \mathrm{MgCl}_{2}$, and $1 \mathrm{mM}$ ATP). To image the individual filaments, $10 \mu \mathrm{L} 10 \mathrm{mM}$ spermidine was incubated for $3.5 \mathrm{~min}$ on a freshly cleaved mica disc glued to a $15 \mathrm{~mm}$ metal specimen disc and then washed three times with $1 \mathrm{~mL}$ of milli-Q water. Without drying the mica, the $900 \mathrm{ng} / \mu \mathrm{L}$ solution of Factin was deposited onto the spermidine-modified mica, incubated for $3.5 \mathrm{~min}$, washed three times with $1 \mathrm{~mL}$ milli-Q water, and then dried with a stream of nitrogen. To get images of bundles, a centrifugation procedure was employed.

Prior to forming the bundles, the F-actin, $\alpha$-actinin, and Caprice proteins were centrifuged for $15 \mathrm{~min}$ at $14,000 \times \mathrm{g}$ at room temperature in a TOMY MX-105 High speed Refrigerated Microcentrifuge (TOMY Tech USA, Inc.) to remove any large aggregates. The bundles were formed by adding $225 \mathrm{ng} / \mu \mathrm{L}$ F-action $(5 \mu \mathrm{M}$ Gactin monomers) and $0.5 \mu \mathrm{M}$ Caprice or $\alpha$-actinin in a final $20 \mu \mathrm{L}$ solution of polymerization buffer and incubated for $30 \mathrm{~min}$ at room temperature. For sample deposition, $500 \mu \mathrm{L}$ of polyacrylamide gel was polymerized in the bottom of a $1.5 \mathrm{~mL}$ microcentrifuge tube. Then a mica disk cut with a hole punch was cleaved and placed in the tube on top of the acrylamide. Then, either $10 \mu \mathrm{L}$ or $1 \mu \mathrm{L}$ of the solution containing the actin bundle was diluted in $200 \mu \mathrm{L}$ of polymerization buffer and deposited in the tube on top of the freshly cleaved mica. The tube was then centrifuged for $15 \mathrm{~min}$ at $14,000 \mathrm{x}$ g with a swing-rotor. The samples were washed by gently dipping the mica disc in milliQ water. The mica was then either air-dried or vacuum-dried before affixing it to a $15 \mathrm{~mm}$ metal specimen disc with double stick tape for imaging.

\subsection{AFM Imaging and Analysis}

AFM images were acquired with the Multimode Atomic Force Microscope, a Nanoscope III Controller, and a Jscanner (Digital Instruments, Inc., USA). Rectangular silicon cantilevers with sharpened tetrahedral tips were used (OMCL AC160TS, Olympus Corp., Japan). These probes had a tip radius of about $7 \mathrm{~nm}$, a resonant frequency of about $300 \mathrm{kHz}$, and a spring constant of about $26 \mathrm{~N} / \mathrm{m}$. The microscope was operated in the Tapping Mode $^{\mathrm{TM}}$ at a scanning rate of $0.5-1 \mathrm{~Hz}$. The images were flattened with the Nanoscope software (v.5.31 r1) and exported as jpegs. The flattened images were analyzed using the Femtoscan software (Advanced Technologies Center, Russia). The heights were measured using the curve tool to trace some of the image background, then along the fibers, and then along some of the background again. This trace was then converted to a cross section to generate a height profile. Profile data was then copied into Microsoft Office Excel (Microsoft Corp., USA) and the average height was calculated over the region(s) of interest. The segmentation in the filament was analyzed by taking the lateral peak-to-peak distance from the height profile of the molecule. All reported errors represent the standard deviation. All surface plots displayed were also generated with the Femtoscan software.

\section{Results}

\subsection{F-Actin Assembly from G-Actin}

In the initial experiments, the samples were prepared using a typical deposition procedure in which either 10 $\mu \mathrm{L} 900 \mathrm{ng} / \mu \mathrm{L}$ F-actin or F-actin/Caprice was deposited on freshly cleaved mica, washed with $3 \mathrm{~mL}$ of water, and then dried by blowing with a stream of nitrogen gas. However, with these methods, the images mostly showed crystals and amorphous material decorating the surface.

Since actin has been reported to be negatively charged at pH 7 [22], a spermidine-modified surface was tried instead. In addition, we wanted to see if more rigorous washing and drying procedures could be used in order to remove the salt crystals observed in the previously described experiments. This method enabled the visualization of individual actin filaments (Figure 1(A)). However, the samples obtained with and without Caprice were nearly identical except that the sample with the Caprice protein added had more background material, likely due to the excess amount of protein in the sample. No bundles could be observed, and there was no clear interaction of Caprice with the fibers, likely due to mechanical disruption of the bundles and the Caprice-fiber interactions or poor adsorption of the bundles to the mica 
surface. In addition, all filaments in the images were oriented in a parallel fashion, suggesting that the actin still does not adhere well to the mica surface even when spermidine is used.

However, this method did provide a way to characterize the morphology of individual actin filaments. The F-actin fibers in Figures 1(A) and (B) had the characteristics segmentation pattern described by other AFM studies of actin [23,24]. A peak-to-peak distance of $33 \pm$ $11 \mathrm{~nm}$ was measured for the F-actin sample. This value corresponds reasonably well to what has been reported in other AFM actin studies [23,25]. A characteristic height profile of an actin filament is displayed in Figure 1(C). The profile depicts a $1.03 \mu \mathrm{m}$ long actin filament with an average height of $1.15 \pm 0.19 \mathrm{~nm}$.

\subsection{From F-Actin Fiber to F-Actin Bundle}

To get images of actin bundles, an alternative deposition procedure was used. Solutions containing preformed actin

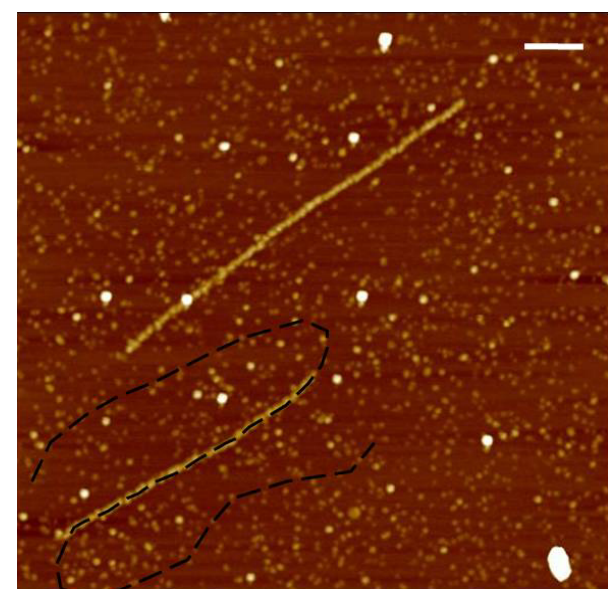

(A)

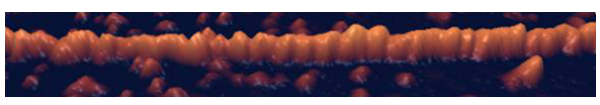

(B)

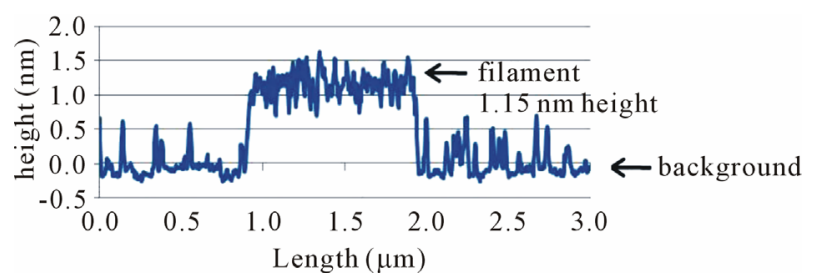

(C)

Figure 1. AFM imaging and analysis of individual actin filaments. (A) A $5 \times 5 \mathrm{\mu m}^{2}$ AFM image of individual actin filaments observed in the F-actin sample without the Caprice bundling protein. Scale bar $=200 \mathrm{~nm}$; (B) A surface plot showing the segmentation pattern of actin filaments observed in the F-actin samples without the Caprice protein; (C) A plot profile of an actin filament produced from the dotted line drawn in (A). bundles formed with either the Caprice or the $\alpha$-actinin protein were centrifuged onto a piece of freshly cleaved mica placed at the bottom of a microcentrifuge tube. The piece of mica was then removed from the tube, gently dipped in water to wash the sample, then vacuum dried. When a $200 \mu \mathrm{L}$ solution containing $0.28 \mathrm{ng} / \mu \mathrm{L}$ of F-actin along with a bundling protein added at a 10:1 actin-protein ratio was centrifuged onto the mica, the images shown in Figures 2(A), 3(A) were obtained. Using this method, we were able to get reasonable concentrations of actin bundles on untreated mica with relatively minimal amounts of crystals or other amorphous material, suggesting that this centrifugation method aided in purification of the bundles.
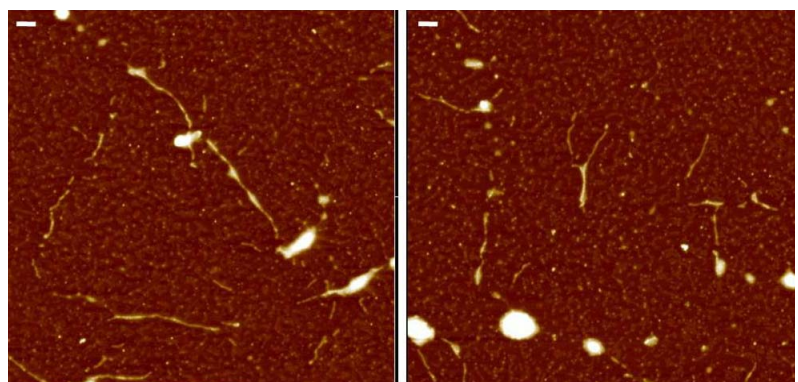

(A)
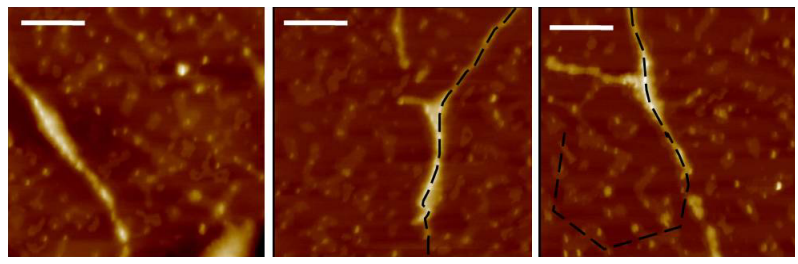

(B)

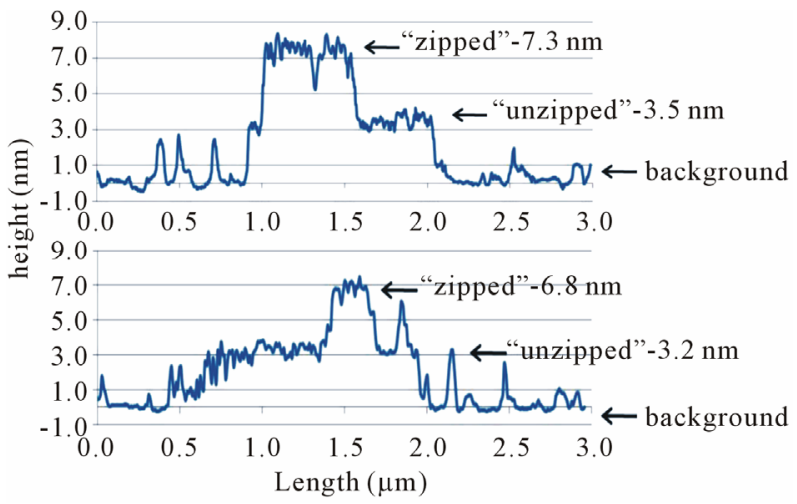

(C)

Figure 2. AFM imaging and analysis of $\alpha$-actinin actin bundle nano-structures. (A) Two representative $5 \times 5 \mu^{2}$ images of the spread of bundle structures on the mica surface; (B) Three $1 \times 1 \mathrm{~m}^{2}$ images showing the "zipper" structures formed with the $\alpha$-actinin protein. All scale bars $=250 \mathrm{~nm}$; (C) Two characteristic plot profiles showing a clear distinction between the height of the "zipped" and "unzipped" regions of the bundle. Parts of the dotted lines used to produce these profiles are shown in (B). 
For the $\alpha$-actinin complexes, zipper-like structures were often observed (Figure 2(B)). Two representative height profiles are shown in Figure 2(C). The heights were bipartite in nature. In these profiles, the area which was "zipped up" had average heights of $7.3 \pm 0.6 \mathrm{~nm}$, and $6.8 \pm 0.4$ for the respective top and bottom profiles, whereas the "unzipped" areas had heights of $3.5 \pm 0.3$ and $3.2 \pm 0.4 \mathrm{~nm}$ for the respective top and bottom profiles. These height values suggest that even the areas of the fibers which appear "unzipped" are already bundled since the height values exceed the height values of the individual filaments measured earlier (Figure 1(C)).

In contrast to the $\alpha$-actinin complexes, Caprice complexes had a much more rigid and highly branched appearance (Figures 3(A) and (B)). In many places the fibers crossed perpendicularly (Figure 3(B)), suggesting that these filaments may have a propensity to form meshwork type structures. Looking at the height profiles of these fibers, they do not have distinct height transitions as observed for the $\alpha$-actinin complexes. The three representative height profiles shown in Figure 3(C) have average heights of $7.0 \pm 1.2 \mathrm{~nm}$ (top), $5.6 \pm 2.2 \mathrm{~nm}$ (middle), and $5.9 \pm 1.2 \mathrm{~nm}$ (bottom). In addition to lacking clear height transitions, the height profiles also have a higher noise level suggesting that the Caprice bundles have some irregularities in the dimensions of the fibers in comparison to the $\alpha$-actinin complexes and individual fibers. This trend is also reflected by the much larger standard deviation values for the average height of the fibers.

When no bundling protein was added to the sample, only an amorphous background with no noticeable actin filaments could be imaged (data not shown). This suggests that the $14,000 \mathrm{x}$ g centrifugation speed which was used is not enough to spin the single filaments out of solution. Additionally, when comparing images of the bundles formed with $\alpha$-actinin and Caprice, the concentration of fibers on the surface appears much larger for the sample with the Caprice protein. This is likely a reflection of the increased bundling efficiency of the Caprice protein. This increased efficiency could be observed with an F-actin bundling assay using an Actin Binding Protein Spin-down Assay Biochem Kit (cosedimentation) from Cytoskeleton Inc. (data not shown).

\subsection{Nano-Meter Scale Analyses of F-Actin Bundles}

When the concentration of protein used for deposition was increased 10 -fold with the same actin-protein ratio maintained, the mica surface was densely coated with networks of F-actin fibers (Figure 4). The F-actin bundles formed with the Caprice protein had a noticeably different morphology than the bundles formed with the
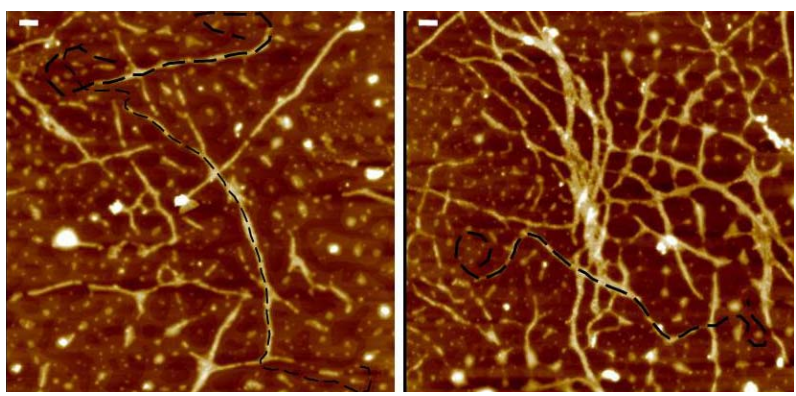

(A)
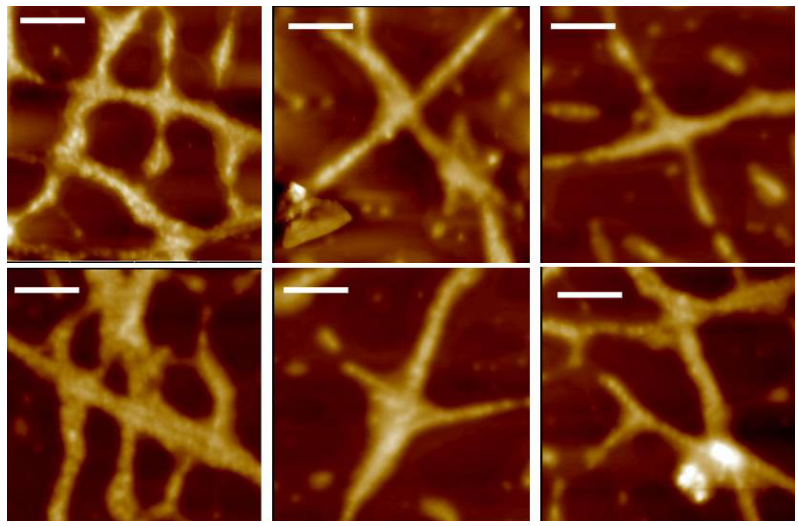

(B)
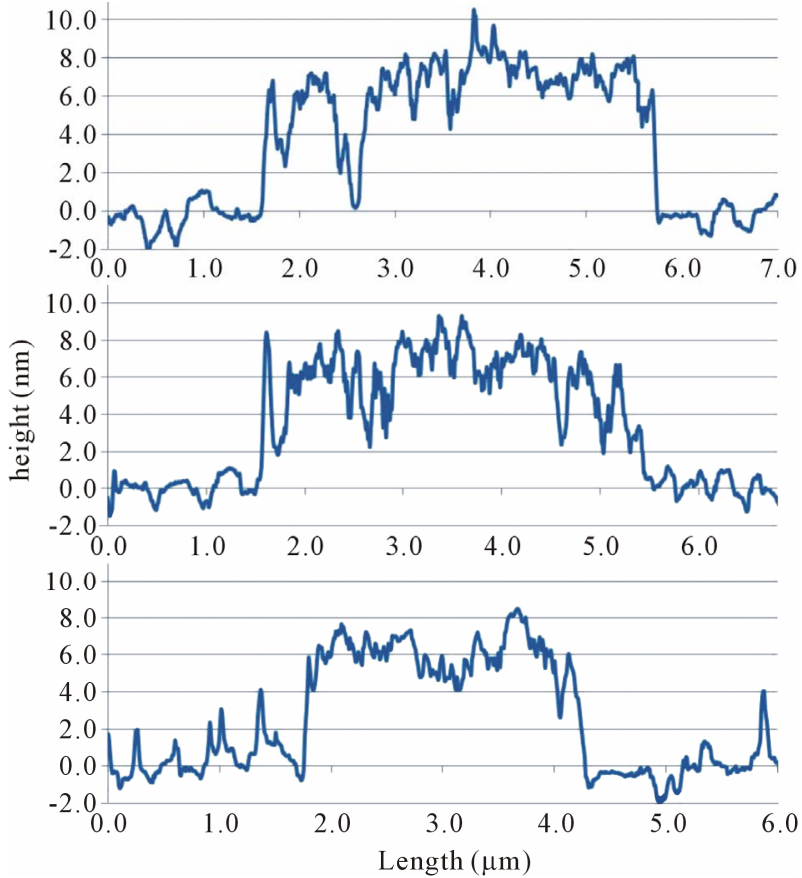

(C)

Figure 3. AFM imaging and analysis of Caprice-actin bundle nanostructures. (A) Two representative $5 \times 5 \mu^{2} \mathrm{im}$ ages of the spread of bundle structures on the mica surface; (B) Six $1 \times 1 \mathrm{\mu m}^{2}$ images showing areas where the fibers crossed perpendicularly. All scale bars $=250 \mathrm{~nm}$; (C) Three characteristic plot profiles showing the height characteristics of the fibers produced from the dotted lines drawn in (A). 


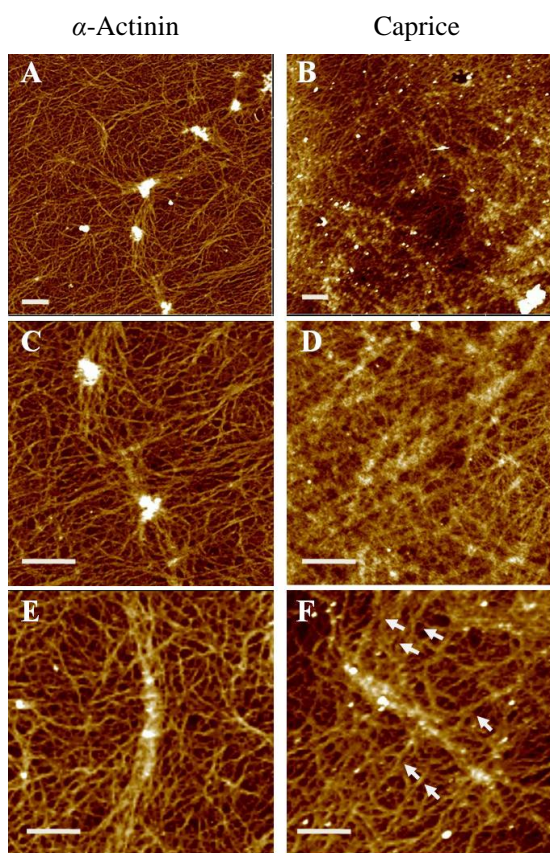

Figure 4. AFM imaging and analysis of actin bundles formed with either the $\alpha$-actinin (A), (C), (E) or the Caprice (B), (D), (F) bundling proteins. (A) $10 \times 10 \mathrm{\mu m}^{2}$ image of the $\alpha$-actinin-actin bundle morphology; (B) $10 \times 10 \mathrm{\mu m}^{2}$ image of the Caprice-actin bundle morphology; (C) $5 \times 5 \mathrm{\mu m}^{2}$ image of a couple of $\alpha$-actinin bundle foci which appear to be at the center of fibers aligned in a parallel orientation; (D) $5 \times$ $5 \mathrm{\mu m}^{2}$ image of compact Caprice-actin bundle structures; (E) Another $5 \times 5 \mathrm{\mu m}^{2}$ image with an $\alpha$-actinin-actin bundle structure with fibers aligned in a parallel orientation with a less distinct focal center; (F) Another $5 \times 5 \mu^{2}$ image with a Caprice-actin bundle structure which appears to have parallelly aligned fibers with additional fibers branching out perpendicularly from it. All scales bars $=1 \mu \mathrm{m}$.

$\alpha$-actinin protein. The actin network containing the Caprice protein had a compact morphology (Figures 4(B) and (D)) whereas the actin network containing the $\alpha$ actinin protein had elongated structures which appeared to be held together by globular foci (Figures 4(A), (C) and $(\mathbf{E})$ ). In regions of the Caprice network which did not appear as dense, elongated structures could also be observed. However, in contrast to the $\alpha$-actinin structures, these also appeared to have perpendicular branches radiating outward from them (Figure 4(F), arrows). When F-actin without a bundling protein was centrifuged onto the mica, this resulted in a surface which appeared amorphous with no observable actin filaments (data not shown). This is unsurprising because in our preliminary experiments, a cosedimentation spin-down assay at the same centrifuge speed showed that actin did not readily localize to the pellet.

\section{Discussion}

In this paper, we have developed a strategy to image ac- tin bundles with AFM by centrifuging the preformed bundles onto a mica surface. Previously, various strategies have been applied to attempts to use AFM for imaging of filamentous actin, including the use of glutaraldehyde fixation or phalloidin to stabilize the filaments [25], the use of lipid monolayers with cationic lipids to attach the actin $[14,26]$, the use of photo-immobilization on azopolymer surfaces [24], and even simple deposition on unmodified mica [23]. The method employed here is based on a F-actin co-sedimentation assay which is frequently used to assess the ability of various actin-binding proteins to form actin bundles [27]. The use of this procedure is allowed for efficient deposition of the bundles in solution onto the mica surface. In addition, the centrifugation speeds used will limit the deposition of single filaments, G-actin, or any non-actin binding proteins in solution, which provide a way to essentially purify the bundles. Moreover, it should be noted that this procedure does not require glutaraldehyde fixation nor chemical modification of the mica surface.

Overall, our method was capable of revealing distinct structures of actin bundles formed by two different actin binding proteins. The $\alpha$-actinin protein is well known to cross-link actin and to bundle it into networks. It has been reported to be involved in focal contacts, stress fiber formations, and muscle cell contraction [28-31]. Structural studies of $\alpha$-actinin-actin bundles have shown that it parallelly cross-links actin filaments, and confocal images have previously shown F-actin to have a bifurcated structure [32,33]. Thus, the zipper structures we observed in this study correspond well to the previously reported effects of $\alpha$-actinin on F-actin bundle structures.

In contrast to $\alpha$-actinin, Caprice is a protein which was recently discovered through proteomic analyses of cellscaffold proteins [19,34]. It was shown to localize to the actin cytoskeleton in HeLa cells. In our images, we observed nanostructures which were perpendicularly crosslinked along with higher order structures with the highly condensed appearance. It is interesting to consider whether the protein is somehow bending the fibers so that they interlink at angles of about $90^{\circ}$, or whether the fibers are simply interwoven. Further examination of the binding mechanism of the Caprice protein to the actin fibers can help to correlate the observed structures to the physiological role of this protein in cells.

In conclusion, our method will be useful to further investigate the molecular mechanisms of F-actin fiber assembly promoted by other actin-binding proteins without the need for any special chemical modifications.

\section{Acknowledgements}

K.T. is supported by a Grant-in-Aid for Scientific Research on Innovative Areas "Molecular basis of host cell competency in virus infection” (\#24115003) from MEXT 
Japan. M.K. is supported by a Grant-in-Aid for Scientific Research on Innovative Areas "Spying minority in biological phenomena” (\#24115512) from MEXT Japan and a Cross-Disciplinary Research Promotion Project from iCeMS, Kyoto University, Japan.

\section{REFERENCES}

[1] P. Hansma, V. Elings, O. Marti and C. Bracker, "Scanning Tunneling Microscopy and Atomic Force Microscopy: Application to Biology and Technology,” Science, Vol. 242, No. 4876, 1988, pp. 209-216. http://dx.doi.org/10.1126/science.3051380

[2] G. Binnig, C. Quate and C. Gerber, “Atomic Force Microscope,” Physical Review Letters, Vol. 56, No. 9, 1986, pp. 930-933. http://dx.doi.org/10.1103/PhysRevLett.56.930

[3] V. Morris, A. Gunning, and A. Kirby, "Atomic Force Microscopy for Biologists,” Imperial College Press, London, 1999. http://dx.doi.org/10.1142/p173

[4] B. Bhushan, "Scanning Probe Microscopy in Nanoscience and Nanotechnology," Springer-Verlag Berlin Heidelberg, Berlin, 2010.

[5] B. Bhushan, "Scanning Probe Microscopy in Nanoscience and Nanotechnology,” Springer-Verlag Berlin Heidelberg, Berlin, 2011.

[6] K. Takeyasu, "Atomic Force Microscopy in Nano-Biology,” Pan Stanford Publishing Co., Singapore City, 2013.

[7] Q. Zhong and D. Inniss, "Fractured Polymer/Silica Fiber Surface Studied by Tapping Mode Atomic Force Microscopy," Surface Science Letters, Vol. 290, No. 1-2, 1993, pp. L688-L692.

[8] K. Hohmura, Y. Itokazu, S. Yoshimura, G. Mizuguchi, Y. Masamura, K. Takeyasu, et al., "Atomic Force Microscopy with Carbon Nanotube Probe Resolves the Subunit Organization of Protein Complexes," Journal of Electron Microscopy (Tokyo), Vol. 49, No. 3, 2000, pp. 415-421. http://dx.doi.org/10.1093/oxfordjournals.jmicro.a023823

[9] J. Gilmore, Y. Suzuki, G. Tamulaitis, V. Siksnys, K. Takeyasu and Y. Lyubchenko, "Single-Molecule Dynamics of the DNA-EcoRII Protein Complexes Revealed with High-Speed Atomic Force Microscopy,” Biochemistry, Vol. 48, No. 44, 2009, pp. 10492-10498. http://dx.doi.org/10.1021/bi9010368

[10] Y. Suzuki, Y. Higuchi, K. Hizume, M. Yokokawa, S. Yoshimura, K. Yoshikawa, et al., "Molecular Dynamics of DNA and Nucleosomes in Solution Studied by FastScanning Atomic Force Microscopy," Ultramicroscopy, Vol. 110, No. 6, 2010, pp. 682-688. http://dx.doi.org/10.1016/j.ultramic.2010.02.032

[11] Y. Suzuki, J. Gilmore, S. Yoshimura, R. Henderson, Y. Lyubchenko and K. Takeyasu, "Visual Analysis of Concerted Cleavage by Type IIF Restriction Enzyme SfiI in Subsecond Time Region,” Biophysical Journal, Vol. 101, No. 12, 2011, pp. 2992-2998.

http://dx.doi.org/10.1016/j.bpj.2011.09.064

[12] H. Sanchez, Y. Suzuki, M. Yokokawa, K. Takeyasu and C. Wyman, "Protein-DNA Interactions in High Speed
AFM: Single Molecule Diffusion Analysis of Human RAD54,” Integrative Biology (Camb), Vol. 3, No. 11, 2011, pp. 1127-1134. http://dx.doi.org/10.1039/c1ib00039j

[13] M. Yokokawa, C. Wada, T. Ando, N. Sakai, A. Yagi, S. Yoshimura, et al., "Fast-Scanning Atomic Force Microscopy Reveals the ATP/ADP-Dependent Conformational Changes of GroEL,” The EMBO Journal, Vol. 25, No. 19, 2006, pp. 4567-4576.

http://dx.doi.org/10.1038/sj.emboj.7601326

[14] N. Kodera, D. Yamamoto, R. Ishikawa and T. Ando, "Video Imaging of Walking Myosin V by High-Speed Atomic Force Microscopy,” Nature, Vol. 468, No. 7320, 2010, pp. 72-76. http://dx.doi.org/10.1038/nature09450

[15] M. Yokokawa and K. Takeyasu, "Motion of the $\mathrm{Ca}^{2+}$ Pump Captured,” FEBS Journal, Vol. 278, No. 17, 2011, pp. 3025-3031. http://dx.doi.org/10.1111/j.1742-4658.2011.08222.x

[16] M. Shibata, T. Uchihashi, H. Yamashita, H. Kandori and T. Ando, "Structural Changes in Bacteriorhodopsin in Response to Alternate Illumination Observed by HighSpeed Atomic Force Microscopy," Angewandte Chemie International Edition, Vol. 50, No. 19, 2011, pp. 44104413. http://dx.doi.org/10.1002/anie.201007544

[17] Y. Suzuki, T. Goetze, D. Stroebel, D. Balasuriya, S. Yoshimura, R. Henderson, et al., "Visualization of Structural Changes Accompanying Activation of N-Methyl-D-Aspartate (NMDA) Receptors Using Fast-Scan Atomic Force Microscopy Imaging," The Journal of Biological Chemistry, Vol. 288, No. 2, 2013, pp. 778-784. http://dx.doi.org/10.1074/jbc.M112.422311

[18] N. Crampton, M. Yokokawa, D. Dryden, J. Edwardson, D. Rao, K. Takeyasu, et al., "Fast-Scan Atomic Force Microscopy Reveals That the Type III Restriction Enzyme EcoP15I is Capable of DNA Translocation and Looping," Proceedings of the National Academy of Sciences of the United States of America, Vol. 104, No. 31, 2007, pp. 12755-12760. http://dx.doi.org/10.1073/pnas.0700483104

[19] Y. Hirano, K. Ishii, M. Kumeta, K. Furukawa, K. Takeyasu and T. Horigome, "Proteomic and Targeted Analytical Identification of BXDC1 and EBNA1BP2 as Dynamic Scaffold Proteins in the Nucleolus," Genes Cells, Vol. 14, No. 2, 2009, pp. 155-166. http://dx.doi.org/10.1111/j.1365-2443.2008.01262.x

[20] M. Kumeta, Y. Hirai, S. Yoshimura, T. Horigome and K. Takeyasu, "Antibody-Based Analysis Reveals 'Filamentous vs. Non-Filamentous' and 'Cytoplasmic vs. Nuclear' Crosstalk of Cytoskeletal Proteins,” Experimental Cell Research, in Press.

[21] M. Zhu, F. Settele, S. Kotak, L. Sanchez-Pulido, L. Ehret, C. Ponting, et al., "MISP Is a Novel Plk1 Substrate Required for Proper Spindle Orientation and Mitotic Progression,” The Journal of Cell Biology, Vol. 200, No. 6, 2013, pp. 773-787. http://dx.doi.org/10.1083/jcb.201207050

[22] K. Zechel and K. Weber, “Actins from Mammals, Bird, Fish and Slime Mold Characterized by Isoelectric Focusing in Polyacrylamide Gels," European Journal of Biochemistry, Vol. 89, No. 1, 1978, pp. 105-112. 
http://dx.doi.org/10.1111/j.1432-1033.1978.tb20901.x

[23] S. Sharma, E. Grintsevich, M. Phillips, E. Reisler and J. Gimzewski, “Atomic Force Microscopy Reveals Drebrin Induced Remodeling of F-Actin with Subnanometer Resolution,” Nano Letters, Vol. 11, No. 2, 2011, pp. 825827. http://dx.doi.org/ 10.1021/nl104159v

[24] T. Ikawa, F. Hoshino, O. Watanabe, Y. Li, P. Pincus and C. Safinya, "Molecular Scale Imaging of F-Actin Assemblies Immobilized on a Photopolymer Surface,” Physical Review Letters, Vol. 98, No. 1, 2007, Article ID: 01801. http://dx.doi.org/10.1103/PhysRevLett.98.018101

[25] Z. Shao, D. Shi and A. Somlyo, "Cryoatomic Force Microscopy of Filamentous Actin," Biophysical Journal, Vol. 78, No. 2, 2000, pp. 950-958. http://dx.doi.org/10.1016/S0006-3495(00)76652-5

[26] D. Shi, A. Somlyo, A. Somlyo and Z. Shao, "Visualizing Filamentous Actin on Lipid Bilayers by Atomic Force Microscopy in Solution," Journal of Microscopy, Vol. 201, No. Pt 3, 2001, pp. 377-382.

[27] R. Meyer and U. Aebi, "Bundling of Actin Filaments by Alpha-Actinin Depends on its Molecular Length," The Journal of Cell Biology, Vol. 110, No. 6, 1990, pp. 20132024. http://dx.doi.org/10.1083/jcb.110.6.2013

[28] S. Ebashi and F. Ebashi, "A New Protein Promoting Contraction of Actomyosin,” Nature, Vol. 203, No. 4945, 1964, pp. 645-646. http://dx.doi.org/10.1038/203645a0
[29] D. Goli, A. Suzuki, J. Temple and G. Holmes, "Studies on Purified-Actinin. I. Effect of Temperature and Tropomyosin on the Actinin-F-Actin Interaction," Journal of Molecular Biology, Vol. 67, No. 3, 1972, pp. 469-488.

[30] O. Pelletier, E. Pokidysheva, L. Hirst, N. Bouxsein, Y. Li and C. Safinya, "Structure of Actin Cross-Linked with Alpha-Actinin: A Network of Bundles,” Physical Review Letters, Vol. 91, No. 14, 2003, Article ID: 148102. http://dx.doi.org/10.1103/PhysRevLett.91.148102

[31] B. Knight, C. Laukaitis, N. Akhtar, N. Hotchin, M. Edlund and A. Horwitz, "Visualizing Muscle Cell Migration in Situ," Current Biology, Vol. 10, No. 10, 2000, pp. 576585. http://dx.doi.org/10.1016/S0960-9822(00)00486-3

[32] J. Ylänne, K. Scheffzek, P. Young and M. Saraste, "Crystal Structure of the Alpha-Actinin Rod Reveals an Extensive Torsional Twist,” Structure, Vol. 9, No. 7, 2001, pp. 597-604. http://dx.doi.org/10.1016/S0969-2126(01)00619-0

[33] B. Sjöblom, A. Salmazo and K. Djinovic-Carugo, "Alpha-Actinin Structure and Regulation," Cellular and Molecular Life Sciences, Vol. 65, No. 17, 2008, pp. 26882701. http://dx.doi.org/10.1007/s00018-008-8080-8

[34] K. Ishii, Y. Hirano, N. Araki, T. Oda, M. Kumeta, K. Takeyasu, et al., "Nuclear Matrix Contains Novel WDRepeat and Disordered-Region-Rich Proteins," FEBS Letters, Vol. 582, No. 23-24, 2008, pp. 3515-3519. 